\title{
Peritubular Myoid Cells Are Not the Migrating Population Required for Testis Cord Formation in the XY Gonad
}

\author{
J. Cool ${ }^{\mathrm{a}}$ F.D. Carmona ${ }^{\mathrm{b}} \quad$ J.C. Szucsik ${ }^{\mathrm{c}} \quad$ B. Capel $^{\mathrm{a}}$ \\ ${ }^{a}$ Department of Cell Biology, Duke University Medical Center, Durham, N.C., USA; \\ ${ }^{b}$ School of Medical Sciences, Institute of Medical Sciences, University of Aberdeen, Aberdeen, UK; \\ 'Division of Developmental Biology, Cincinnati Children's Hospital Medical Center, Cincinnati, Ohio, USA
}

\section{Key Words}

Migration · Peritubular myoid cells · Sex determination •

Testis cords

\begin{abstract}
Cell migration is one of the earliest events required for development of the testis. Migration occurs only in XY gonads downstream of Sry expression and is required for the subsequent epithelialization of testis cords. Using organ culture experiments and tissue recombination, we and others speculated that peritubular myoid (PTM) cells were among the migratory cells and were likely the cell type required for cord formation. However, because no unique marker was found for PTM cells, their positive identification during or after migration remained unclear. $\alpha$-Smooth Muscle Actin ( $\alpha$ Sma; approved gene symbol Acta2), a classic marker of adult PTM cells, is expressed broadly in testis interstitial cells at E12.5, and becomes highly enriched in PTM cells by E15.5-16.5. We used a novel transgenic line expressing EYFP under the control of an $\alpha S m a$ promoter to determine whether $\alpha S m a-E Y F P$ positive cells migrate into the gonad. Surprisingly, mesonephroi expressing $\alpha S m a-E Y F P$ do not contribute any EYFP positive cells to $\mathrm{XY}$ gonads when used as donors in recombination cultures. These results indicate that $\alpha$ Sma-EYFP cells do not migrate into the gonad during the critical window of sex determination and cannot be the migrating cell
\end{abstract}

type required for testis cord formation. Our results suggest that PTM cells, and most other interstitial lineages, with the exception of endothelial cells, are induced within the gonad. These experiments suggest that endothelial cells are the migrating cell type required for epithelialization of testis cords.

Copyright $\odot 2008$ S. Karger AG, Basel

The epithelialization of Sertoli cells and morphogenesis of seminiferous tubules has been a primary focus of the sex determination field for many years. Classic experiments initiated by Skinner, Fritz, and Tung sought to identify any factors, hormones, or cell interactions that are required for morphogenesis of testis cords. A Sertoli cell fraction was isolated by dissociation and centrifugation of adult rat testes. Additional fractions and factors were added to the Sertoli cultures to identify conditions that increased epithelialization of Sertoli cells. Interestingly, the addition of purified stromal cells to cultures increased matrix deposition and the appearance of cordlike epithelial structures [Tung and Fritz, 1980; Skinner et al., 1985]. The squamous shape of the stromal fraction suggested a myoid identity, although a positive identification was never made. A role for peritubular myoid cells (PTM) during Sertoli cell epithelialization was attractive because these cells line the outside of seminiferous tu-

\section{KARGER}

Fax +4161306 1234

E-Mail karger@karger.ch

www.karger.com
(C) 2008 S. Karger AG, Basel

1661-5425/08/0023-0128\$24.50/0

Accessible online at:

www.karger.com/sxd
Dr. Blanche Capel

Department of Cell Biology, Duke University Medical Center, Box 3471

Genome Science Research Building II, Room 4026, Research Drive

Duke University, Durham, NC 27710 (USA)

Tel. +1 919684 6390, Fax +1 919668 3467, E-Mail b.capel@cellbio.duke.edu 
bules and could partner with Sertoli cells during testis cord formation.

Subsequently, organ culture tissue recombination experiments revealed a wave of sex-specific cell migration from the mesonephros into the XY gonad, and established that cells entering the gonad are required for morphogenesis of seminiferous tubules [Buehr et al., 1993; Merchant-Larios et al., 1993; Tilmann and Capel, 1999]. Histological sections of these organ recombinations showed that cells entering the gonad were squamous cells immediately adjacent to Sertoli cells [Martineau et al., 1997]. In the context of the previous studies on adult and neonatal rodents, these studies led to the conclusion that PTM cells migrate into the male gonad where they are required for Sertoli cell epithelialization.

At the time of the initial migration experiments, few cell-specific markers were available to identify migrating populations. It was shown that most cells that enter the testis are positive for the endothelial marker PECAM-1, consistent with the establishment of a de novo arterial network by E12.5 in the testis [Brennan et al., 2002]. PECAM-1 negative cells were rare and were not identified by any specific marker. Most of these unidentified cells were located adjacent to cord boundaries and were assumed to be PTM cells based on earlier work [Martineau et al., 1997]. Another group sorted migrating cells, and after a period of culture in vitro, reported that cultured cells gave rise to several lineages including PTM cells [Nishino et al., 2001]. Nonetheless, on-going efforts to identify a specific marker for PTM cells in vivo were unsuccessful [Jeanes et al., 2005], thus the question of whether these cells were actually part of the migrating population remained open.

In adult muscle, various actin isoforms are frequently specific to particular populations of myoid cells. However, during development many myoid markers are expressed across cell types and become specific subsequently. Antibody staining of $\alpha$ SMA (Acta2) showed that 2-3 days after sex determination, it is expressed throughout the interstitium and enriched in PTM cells in rodent testes [Palombi et al., 1992; unpublished data of B.C.]. However, during the fetal stages when migration can be assayed (E11.5-E13.5) $\alpha S m a$ is broadly expressed at a low level in the mesonephros and in most interstitial cells of the testis. For this reason, antibodies were not informative when used on recombination assays to specifically identify PTM cells from among the migrating population.

Endothelial cells represented a large proportion of the migrating cell population. However, at the time of the original experiments, no function other than nutrient and gas exchange had been ascribed to the endothelium. Subsequently, endothelial cells were shown to affect the development and differentiation of surrounding tissues independent of circulation [Lammert et al., 2001; Matsumoto et al., 2001]. This raised the possibility that endothelial cells entering the testis from the mesonephros, rather than PTM cells, are responsible for the induction of testis cords, which led us to readdress the question of whether PTM cells are part of the migrating population.

To address this question, we utilized the advantages of in vivo analysis with a new transgenic mouse line, which expresses EYFP (enhanced yellow fluorescent protein) under the control of the $\alpha S m a$ promoter $(\alpha S m a-E Y F P)$. In this transgenic mouse, EYFP is expressed brightly and broadly in the mesonephros and in most interstitial cells of the gonad, including PTM cells, from $\sim$ E12.5. We reasoned that the broad expression of $\alpha$ Sma-EYFP could be advantageous. Even though $\alpha S m a-E Y F P$ is not specific to PTM cells, recombination cultures using an $\alpha S m a-E Y F P$ mesonephros and a wild type gonad could reveal whether any EYFP-positive interstitial cells (including PTM cells) migrate from the mesonephros into the gonad. We show that no $\alpha S m a-E Y F P$ positive cells migrate from the mesonephros during the period when migration is required for testis cord formation to occur.

\section{Materials and Methods}

Mouse Strains, Matings, and Tissue Recombination WT and $\alpha S m a-E Y F P$ mice were maintained on an outbred CD-1 background while EGFP (Tg(GFPU)5Nagy) mice were maintained on FVB. Mice were checked daily and E0.5 was set as noon on the day a vaginal plug was detected. Amnion stains were used to determine the sex of the embryo by the detection of condensed sex chromatin bodies (Barr bodies) in XX individuals as described [Palmer and Burgoyne, 1991]. All genotypes were confirmed by PCR. Whole genital ridges were removed and separated from sexed embryos. After separation, WT XY gonads and transgenic mesonephroi were recombined on agar blocks as previously described [Martineau et al., 1997]. All cultures were then incubated under previously described conditions for 24-48 h.

\section{Immunohistochemistry}

Whole mount immunohistochemistry was performed as previously described [Brennan et al., 2002]. Antibodies were rat antiPECAM-1 (Pharmingen; 1:500 dilution) and rabbit anti-laminin (Kind gift of Harold Erickson; 1:250 dilution). Confocal optical Z-sections ( $512 \times 512$ pixel arrays) were collected on a Zeiss LSM 510 META confocal microscope. Maximum intensity projections were created using Zeiss 510 META confocal software. 
Fig. 1. $\alpha$ Sma-EYFP labels a subset of interstitial cells including peritubular myoid cells. A Optical confocal Z-sections of $\alpha S m a-E Y F P$ testes. EYFP is expressed throughout the interstitium but never within testis cords. At early (E12.5) and late (E15.5) stages of testis development, EYFP is enriched in cells immediately adjacent to the laminin (red) rich extracellular matrix (arrowheads). A' Magnification of a small portion of $\mathbf{A}$, as outlined by the white box, shows $\alpha$ Sma-EYFP (green) expressed in presumptive peritubular cells immediately adjacent to the ECM (red). B High magnification images of $\alpha S m a-$ EYFP gonads demonstrate that expression is excluded from PECAM-1 (red) positive endothelial cells (arrowheads).
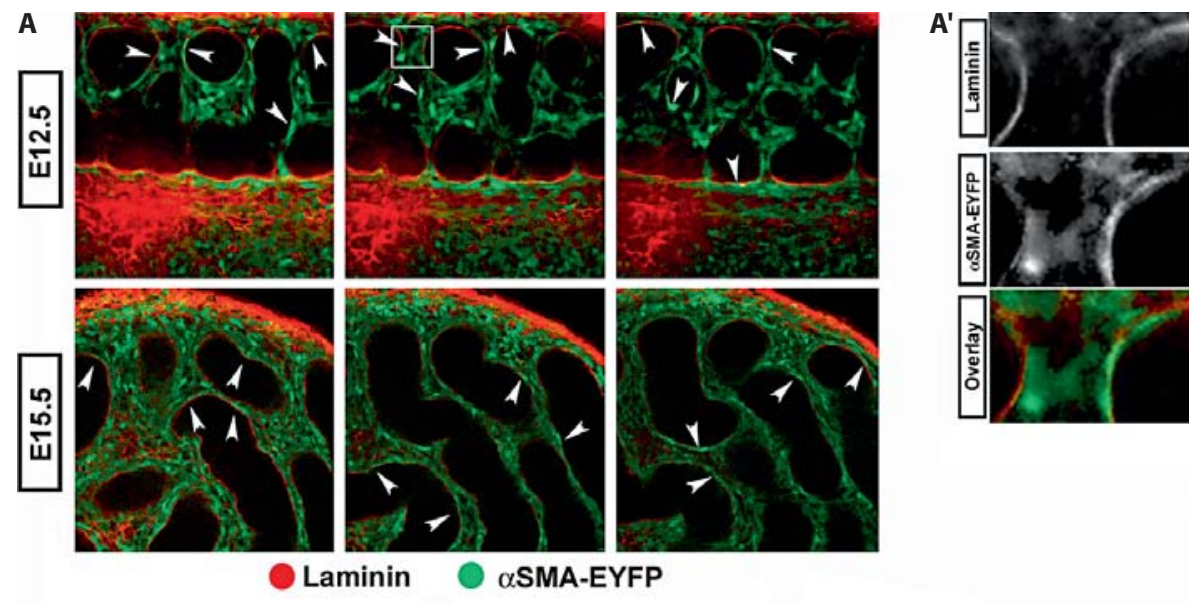

B

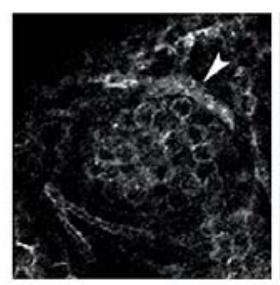

PECAM-1

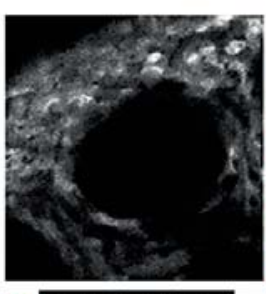

QSMA-EYFP

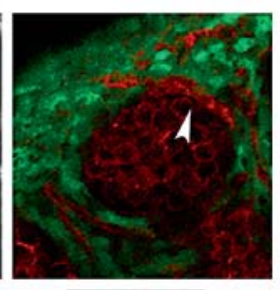

Overlay

\section{Results}

aSma-EYFP Is Enriched in Peritubular Myoid Cells

To characterize the expression of the $\alpha S m a-E Y F P$ transgene in the testis, we examined gonads at stages between E11.5 and E18.5 using confocal microscopy. $\alpha$ Sma$E Y F P$ was never seen within testis cords but was expressed in many interstitial cells of the testes (fig. 1A). Expression of the transgene did not result in developmental delays or altered fertility. Importantly for the purpose of this work, $\alpha S m a-E Y F P$ was not expressed in endothelial cells (fig. 1B), but was enriched in the squamous PTM cells adjacent to Sertoli cells at both E12.5 and E15.5 (fig. 1A, arrowheads), and at later stages. Higher magnification images show EYFP enriched cells are immediately adjacent to the laminin rich extracellular matrix surrounding testis cords confirming proper PTM localization (fig. $1 A^{\prime}$ ).

$\alpha$ Sma-EYFP shows clear expression in PTM and other interstitial cells and therefore serves as an effective marker for many cells in the interstitial compartment of the testis with the exception of endothelial cells. Expression of the EYFP protein in this transgenic model was robust and stable such that no antibodies were needed for its de- tection after fixation or during prolonged live imaging. This preliminary data suggested that $\alpha S m a-E Y F P$ would represent a robust marker for tissue recombination experiments designed to distinguish between the migration of endothelial cells and the migration of other interstitial cell types, including PTM cells.

\section{$\alpha$ Sma-EYFP Positive Cells Do Not Migrate from the Mesonephros}

Migration from the mesonephros into the XY gonad was originally detected using tissue recombinations. Recombinations are composed of wild-type gonads that are removed from the adjacent mesonephros and recombined with a mesonephros expressing a transgenic label ( $\beta$-gal or EGFP). To assay general migration of mesonephric cells to the gonad, recombinations with a mesonephros expressing a ubiquitous marker were performed. To assay the migration of a single lineage, recombinations with mesonephroi expressing cell-specific markers were performed. Assays designed to detect endothelial migration revealed robust vascular migration into the gonad and confirmed the arterial identity of migrating cells [Brennan et al., 2002]. To determine whether myoid cells migrate into the testis, we recombined $\alpha S m a-E Y F P$ me- 
Fig. 2. $\alpha S m a-E Y F P$ positive cells do not migrate into the testis. Control experiments confirmed robust migration of cells from the mesonephros $(\mathrm{m})$ into the gonad (g) at E11.5 (A) and E12.5 (B). As previously reported, the vast majority of these cells co-label with the endothelial marker PECAM-1 (C). Identical recombinations using $\alpha$ Sma-EYFP mesonephroi resulted in no migration when initiated at E11.5 (D) and E12.5 (E). In these experiments migration and testis cord formation occurred normally as indicated by PECAM-1 endothelial cells (arrowheads) and germ cell aggregation (asterisks). All images are maximum intensity projections of multiple optical sections. Migration of $\alpha S m a-E Y F P$ cells was never observed whereas panEGFP controls nearly always contributed cells to wild type XY gonads (F).
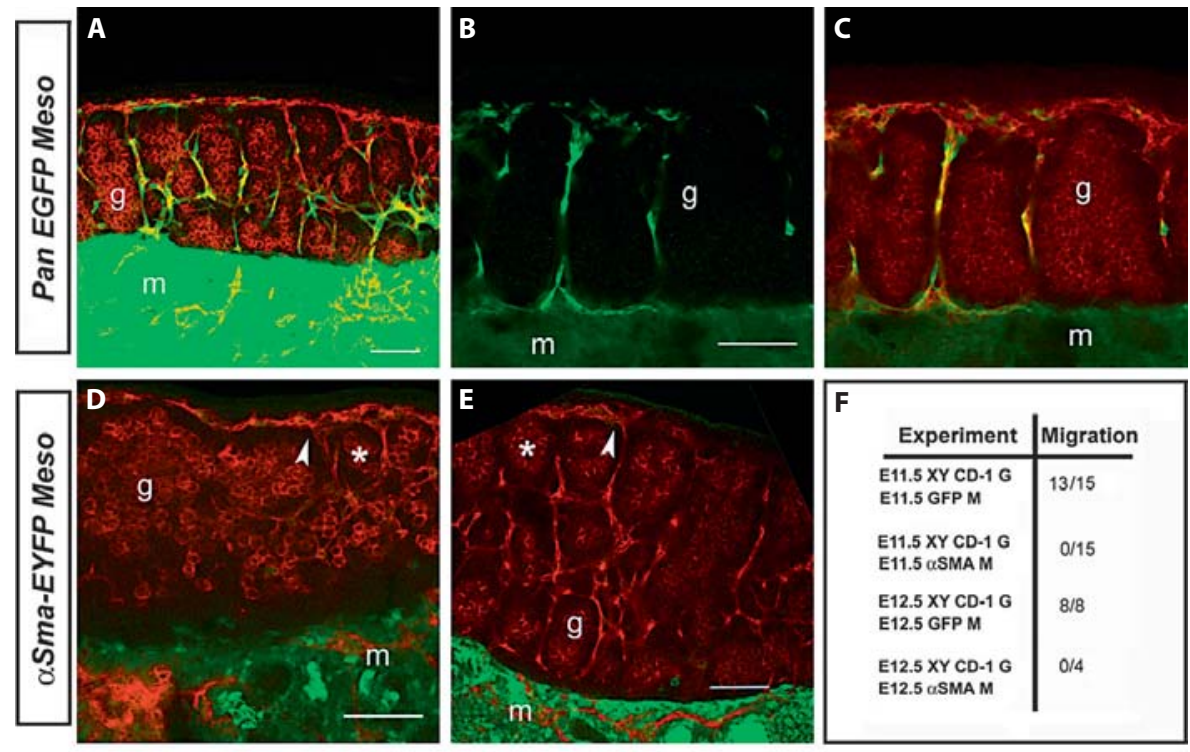

PECAM-1 sonephroi with wild type CD-1 XY gonads. Recombinations were initiated at or prior to E11.5 and cultured for 24-36 h.

In parallel with mesonephroi expressing $\alpha S m a-E Y F P$, a ubiquitous EGFP expressing transgenic line was used as a positive control. When recombined with $\mathrm{XY}$ gonads, cells from EGFP mesonephroi migrated into $\mathrm{XY}$ gonads as previously described (fig. 2A-C, F). Live imaging of EGFP recombinations showed migration of endotheliallike cells to the coelomic domain of the testis. Cells entering the gonad extended long filopodial extensions that appeared to direct their migration toward the coelomic epithelium where they established the presumptive male vasculature [see also Coveney et al., 2008]. In contrast, when using an $\alpha S m a-E Y F P$ mesonephros as a donor, EYFP positive cells were never found in recombinations with CD-1 XY gonads (fig. 2D-F). In $\alpha S m a-E Y F P$ recombinations, testis cords (asterisks) formed and PECAM-1 positive endothelial cells (arrowheads) were still detected (fig. 2D, E) suggesting that endothelial migration and cord formation occurred normally. Although many recombinations were initiated as early as E11.25, we never observed $\alpha$ Sma-EYFP expressing cells within the gonad.

A possible explanation for the observed lack of migration is that the PTM cells reside only in XY mesonephroi. Previous work did not detect a difference based on the XX or XY genotype of the mesonephros [Martineau et al., 1997]. However, we could not rule out the migration of a smaller population of cells present only in XY mesoneph- roi and masked by the robust migration of endothelial cells. To address this possibility, we assembled recombinations in which both $\alpha$ Sma-EYFP mesonephroi and CD-1 gonads came from XY embryos. These experiments confirmed our earlier results: $\alpha S m a-E Y F P$ expressing cells do not migrate from the mesonephros into the testis between E11.5-E12.5.

The absence of migrating EYFP positive cells in the E11.5 $\alpha$ Sma-EYFP recombination experiments indicates that PTM cells do not migrate during the bipotential window and are unlikely to be the migrating population required for testis cord formation. However, the possibility remained that PTM cells entered the gonad after the bipotential window and were found in previous recombinations due to their migration after testis cords had formed. Accordingly, we initiated recombination cultures at stages between E12.5 and E13.5. Recombinations initiated at E12.5 with mesonephroi expressing ubiquitous EGFP showed robust migration restricted to interstitial cells of the testis (fig. $2 \mathrm{~B}$ ). In contrast, $\alpha S m a-E Y F P$ mesonephroi did not contribute EYFP positive cells to CD-1 gonads despite normal development of the testis over the culture period (fig. 2E). Subsequent staining indicated that cells entering the testis between E12.5 and E13.5 were PECAM-1 positive endothelial cells, consistent with earlier studies of endothelial migration between E11.5 and E13.5 (fig. 2C).

Overall, we never observed EYFP positive cells entering XY gonads after recombination with $\alpha S m a-E Y F P$ 
mesonephroi at E11.5 or E12.5 (0/19). This was in stark contrast to migration observed in nearly all recombinations using ubiquitous EGFP mesonephroi and XY gonads $(21 / 23$, fig. $2 \mathrm{~F})$.

\section{Discussion}

In vitro culture of adult Sertoli cells demonstrated that squamous stromal cells induced epithelialization of cordlike structures and establishment of a basal lamina. Furthermore, mesonephric migration into the gonad between E11.5 and E12.5 was shown to be required during epithelial morphogenesis of testis cords [Buehr et al., 1993; Tilmann and Capel, 1999]. A subset of migrating cells was found to be squamous cells located near testis cord boundaries. Combined, these findings gave rise to the idea that PTM cells migrate into the testis from the mesonephros and actively participate in testis cord formation. However, in both of these studies PTM cells were never positively identified using molecular markers. Conclusions were based on stromal localization and squamous morphology of the migrating cells.

In an effort to positively identify migrating cells and confirm previous conclusions, we utilized an $\alpha$ Sma-EYFP transgene that efficiently labels both PTM cells and other interstitial populations. Expression of the transgene, albeit non-specific, is nonetheless an effective label for PTM cells at early stages of testis development. As $\alpha S m a-$ EYFP was not expressed in endothelial cells, their migration could be separated from PTM and other interstitial cells. Thus, we concluded that $\alpha S m a-E Y F P$ mesonephroi would clarify previous recombination experiments by distinguishing endothelial and PTM cell migration. Recombinations using $\alpha S m a-E Y F P$ and EGFP positive controls showed that under culture conditions where robust migration occurs, neither PTM cells nor any other EYFP positive cell type enters the gonad from the mesonephros. Although recombinations were performed throughout and beyond the bipotential window, $\alpha S m a-E Y F P$ expressing cells were never observed in the XY gonad, despite the fact that testis cord formation occurred normally. While it is conceivable that these $\alpha S m a-E Y F P$ transgenic cells are disadvantaged in some way, it seems unlikely based on the normal development of $\alpha S m a-$ EYFP transgenic testes. In any case, this experiment proves that migration of PTM cells is not required for male morphogenesis, as this process occurred normally in the absence of PTM migration. It is important to note that these results do not refute a requirement for PTM cells during testis cord formation, but suggest that PTM cells are induced within the testis, at least in the mouse.

Based on these results, PECAM-1 positive endothelial cells are the only identified population that enters the testis. Previously, a small population of PECAM-1 negative cells was detected after recombination with mesonephroi expressing a ubiquitous marker [Martineau et al., 1997]. At present we cannot account for these cells, but they were a rare minority population relative to the numbers of endothelial cells detected in these assays.

Membrane barriers placed between the mesonephros and gonad during recombination culture resulted in disruption of testis cord formation [Buehr et al., 1993; Tilmann and Capel, 1999]. These assays confirmed the requirement for cell migration but did not clarify which cell population is required. Our present results suggest that reassessing endothelial migration may clarify the influence of the endothelium during testis cord morphogenesis. Endothelial cells are responsible for cell differentiation and patterning in both the liver and pancreas raising the possibility of a similar function in the testis [Lammert et al., 2001; Matsumoto et al., 2001; Coveney et al., 2008]. Future work must selectively inhibit individual populations of cells to determine which cell type(s) are required for the epithelialization of testis cords.

\section{Acknowledgement}

We thank Dr. James Lessard (Cincinnati Childrens Hospital) for bringing the $\alpha S m a-E Y F P$ line to our attention and providing the mice.

\footnotetext{
References Brennan J, Karl J, Capel B: Divergent vascular mechanisms downstream of Sry establish the arterial system in the XY gonad. Dev Biol 244:418-428 (2002).

Buehr M, Gu S, McLaren A: Mesonephric contribution to testis differentiation in the fetal mouse. Development 117:273-281 (1993).

Coveney D, Cool J, Oliver T, Capel B: Four dimensional analysis of vascularization during primary development of an organ, the gonad. Proc Natl Acad Sci USA 105:72127217 (2008).

- Jeanes A, Wilhelm D, Wilson MJ, Bowles J, McClive PJ, et al: Evaluation of candidate markers for the peritubular myoid cell lineage in the developing mouse testis. Reproduction 130:509-516 (2005).

Lammert E, Cleaver O, Melton D: Induction of pancreatic differentiation by signals from blood vessels. Science 294:564-567 (2001).
} 
Martineau J, Nordqvist K, Tilmann C, LovellBadge R, Capel B: Male-specific cell migration into the developing gonad. Curr Biol 7: 958-968 (1997).

-Matsumoto K, Yoshitomi H, Rossant J, Zaret KS: Liver organogenesis promoted by endothelial cells prior to vascular function. Science 294:559-563 (2001).

-Merchant-Larios H, Moreno-Mendoza N, Buehr $\mathrm{M}$ : The role of the mesonephros in cell differentiation and morphogenesis of the mouse fetal testis. Intl J Dev Biol 37:407-415 (1993).
Nishino K, Yamanouchi K, Naito K, Tojo H: Characterization of mesonephric cells that migrate into the XY gonad during testis differentiation. Exp Cell Res 267:225-232 (2001).

Palmer SJ, Burgoyne PS: The Mus musculus domesticus Tdy allele acts later than the Mus musculus musculus Tdy allele: A basis for XY sex reversal in C56Bl/6-Y ${ }^{\mathrm{POS}}$ mice. Development 113:709-714 (1991).

Palombi F, Farini D, Salanova M, de Grossi S, Stefanini M: Development and cytodifferentiation of peritubular myoid cells in the rat testis. Anat Rec 233:32-40 (1992).
Skinner MK, Tung PS, Fritz IB: Cooperativity between Sertoli cells and testicular peritubular cells in the production and deposition of extracellular matrix components. J Cell Biol 100:1941-1947 (1985).

Tilmann C, Capel B: Mesonephric cell migration induces testis cord formation and Sertoli cell differentiation in the mammalian gonad. Development 126:2883-2890 (1999).

Tung PS, Fritz IB: Interactions of Sertoli cells with myoid cells in vitro. Biol Reprod 23: 207-217 (1980). 